\title{
N7-methylguanosine modification of IncRNAs in a rat model of hypoxic pulmonary hypertension: a comprehensive analysis
}

\author{
Huan Wang ${ }^{1 \dagger}$, Ren Biao Chen ${ }^{2 \dagger}$, Si Ni Zhang ${ }^{1}$ and Rui Feng Zhang ${ }^{1 *}$
}

\begin{abstract}
Background: Long non-coding RNAs (IncRNAs) play a critical role in the pathogenesis of hypoxic pulmonary hypertension (HPH). The role of N7-methylguanosine (m7G) modification in IncRNAs has received increased attentions in recent years. However, the m7G-methylation of IncRNA in HPH has yet to be determined. We have therefore performed a transcriptome-wide analysis of $m 7 \mathrm{G}$ IncRNAs in $\mathrm{HPH}$.

Results: Differentially-expressed m7Gs were detected in $\mathrm{HPH}$, and $\mathrm{m} 7 \mathrm{G}$ IncRNAs were significantly upregulated compared with non-m7G IncRNAs in HPH. Importantly, this was the first time that the upregulated m7G IncXR_591973 and $\mathrm{m7G}$ IncXR_592398 were identified in HPH.

Conclusion: This study provides the first m7G transcriptome-wide analysis of HPH. Importantly, two HPH-associated m7G IncRNAs were identified, although their clinical significance requires further validation.
\end{abstract}

Keywords: Hypoxic pulmonary hypertension, IncRNAs, N7-methylguanosine modification, m7G-sequencing

\section{Introduction}

Pulmonary hypertension $(\mathrm{PH})$ is a lethal disease causing increased vascular resistance and eventually leading to right ventricular failure and death [1]. Hypoxic pulmonary hypertension (HPH) is classified as a group III PH based on pathogenesis. It is a progressive disease induced by chronic hypoxia, which normally results from severe interstitial lung diseases and chronic obstructive pulmonary disease (COPD) [2]. The pathophysiology of $\mathrm{HPH}$ is characterized by pulmonary arterial remodelling, mediated via unusual proliferation of pulmonary artery endothelial cells (PAECs) and pulmonary artery smooth muscle cells (PASMCs), but also activation of quiescent

\footnotetext{
*Correspondence: zrf1977313@yeah.net

†Huan Wang and Ren Biao Chen contributed equally to this work.

${ }^{1}$ Department of Respiratory Medicine, Zhongda Hospital of Southeast

University, 87 Dingjiaqiao Road, Nanjing 210009, People's Republic

of China

Full list of author information is available at the end of the article
}

fibroblasts [3, 4]. However, the underlying molecular mechanisms remain imperfectly understood due to the complexity and malignancy of $\mathrm{HPH}$.

In recent years, long non-coding RNAs (lncRNAs) have received extensive attention because of their critical role in the regulation of biological phenomena such as cell proliferation, apoptosis, migration and invasion [5-8]. IncRNAs generally refer to a class of transcripts longer than 200 nucleotides (nt) that lack protein-coding ability [9]. They interact with proteins, interfere with microRNA by acting as molecular sponges, modify the epigenome and interfere with gene expression by binding with gene promoters [1013]. The crucial role of lncRNAs in HPH pathogenesis in idiopathic $\mathrm{PH}$ was highlighted by the up-regulation of 2004 lncRNAs and down-regulation of 507 lncRNAs [14]. Subsequently, multiple lncRNAs have been implicated in the pathogenesis of HPH, such as lncRNA MEG3 and lncRNA Tug1. It is noteworthy that their dysregulated expression has been observed in HPH [15-17]. RNA modification 
plays a critical role in the regulation of gene expression [18]. Nevertheless, post-transcription modification of lncRNAs in HPH remains largely unexplored.

N7-Methylguanosine (m7G) is a ubiquitous post-transcriptional modification of mRNA and lncRNA in eukaryotes, and it is essential for efficient gene expression and cell viability $[19,20]$. During transcription, $\mathrm{m} 7 \mathrm{G}$ is incorporated at the $5^{\prime}$ end, modulating the various events in the mRNA cycle including RNA splicing, polyadenylation, nuclear export and translation [21]. Although evidence increasingly indicates that $\mathrm{m} 7 \mathrm{G}$ modification is closely associated with the initiation and progression of various diseases, the RNA m7G-methylation profile of HPH has yet to be reported. This study provides the first transcriptomewide analysis of $\mathrm{m} 7 \mathrm{G}$ profile in $\mathrm{HPH}$ and control group, demonstrating the enormous diversity of $\mathrm{M} 7 \mathrm{G}$ modification patterns in these two groups. It is hoped that this study will facilitate further investigation into the potential role of m7G modification in $\mathrm{HPH}$ pathogenesis.

\section{Results}

\section{General features of $\mathrm{m7G}$ methylation of IncRNAs in HPH} and control samples

A rat model of hypoxia-induced $\mathrm{PH}$ was successfully established [22]. Pulmonary tissues from HPH and control (normoxia, $\mathrm{N}$ ) rats were used for transcriptome-wide m7G-MeRIP-seq and RNA-seq analyses. The HPH tissues revealed 2685 m7G peaks, while the control rat tissues showed $2644 \mathrm{~m} 7 \mathrm{G}$ peaks. The HPH and control tissues shared 255 peaks, which accounted for only $5.0 \%$ of all peaks in the two groups (Fig. 1a; Additional file 1: Data S1). The low percentage of shared $\mathrm{m} 7 \mathrm{G}$ peaks within the lncRNAs indicated differences in the 7 7G pattern between the two groups. Additionally, the level of $\mathrm{m} 7 \mathrm{G}$ in the total lncRNA derived from $\mathrm{HPH}$ rats was lower than that in the control group (Fig. 1b).

Analysis of the distribution of $\mathrm{m} 7 \mathrm{G}$ peaks in each lncRNA revealed no significant difference between the two groups. Approximately $80 \%$ of the modified lncRNAs showed unique $\mathrm{m} 7 \mathrm{G}$ peaks, while almost $10 \%$ of the modified lncRNAs revealed two m7G peaks (Fig. 1c).

To further elucidate the $\mathrm{m} 7 \mathrm{G}$ methylation pattern of lncRNAs in the HPH and control groups, the samples were divided into the following six categories based on the positional relationship between the m7G methylated lncRNAs and mRNAs: bidirectional, exon sense overlap, intron sense overlap, intron antisense overlap, natural antisense overlap, and intergenic overlap. The results revealed that the majority of lncRNAs contained m7G sites with exon sense overlap. In deed, $\mathrm{HPH}$ samples accounted for $53 \%$, while control samples constituted $54 \%$ of such lncRNAs. Most lncRNAs carried intergenic m7G sites, with HPH samples accounting for $23 \%$ and control samples constituting $25 \%$ of such lncRNAs. Only approximately $2 \%$ of the methylation sites were located in the intronic antisense group (Fig. 1d-e).

Further analysis of the m7G methylated lncRNAs showed that more than $30 \%$ of total lncRNA contained two exons in both HPH and control groups (Fig. 1f-g). Similarly, m7G and non-m7G lncRNAs were mainly encoded by two exons, suggesting that the two exons in the HPH and control groups contributed to most of the m7G methylation in IncRNAs.

\section{Length and distribution of differentially-methylated IncRNAs}

As displayed in the heatmap of m7G lncRNA expression (Fig. 2a), 353 differentially methylated $\mathrm{m} 7 \mathrm{G}$ sites were identified across 344 lncRNAs. Of these, 55\% (193/353) were significantly hypermethylated and 45\% (160/353) were significantly hypomethylated (fold change $\geq 2$ and $P \leq 0.00001$; Fig. 2b; Additional file 2: Data S2). The 353 differentially methylated $\mathrm{m} 7 \mathrm{G}$ sites were located in 344 lncRNAs, including hypermethylated (189) and hypomethylated (155) types (Fig. 2b).

Analysis of the length of the differentially m7G-methylated lncRNAs revealed differences between hypermethylated and hypomethylated types (Fig. 2c). The length of the hypermethylated $\mathrm{m} 7 \mathrm{G}$ IncRNAs was primarily 1-1000 bp (24\%) and 2000-3000bp (23\%), while the length of the hypomethylated $\mathrm{m} 7 \mathrm{G}$ lncRNAs was mostly in the range of 1000 to $2000 \mathrm{bp}$ (29\%).

To analyse the distribution of the differentially m7Gmethylated lncRNAs across chromosomes, the enrichment level of m7G methylated lncRNAs on each chromosome was further determined. As shown in Fig. 2d, the hypomethylated $\mathrm{m} 7 \mathrm{G}$ lncRNAs were primarily located on chromosomes 1 (13.99\%), 5 (7.25\%) and 4 (6.74\%) and the hypomethylated m7G lncRNAs were primarily located on chromosomes 1 (15.63\%), 10 (10.0\%) and $3(6.88 \%)$.

\footnotetext{
(See figure on next page.)

Fig. 1 Overview of $\mathrm{m} 7 \mathrm{G}$ methylation within IncRNAs in the lungs of HPH and normobaric normoxic rats. a Venn diagram showing the number of HPH-unique, normoxic (N)-unique and common m7G peaks of IncRNAs. b Boxplot of m7G peaks enrichment in IncRNAs of HPH and N groups. $\mathbf{c}$ Proportion of IncRNAs with different numbers of $\mathrm{m7G}$ peaks in the two groups. $\mathbf{d}$ and e Classification of total IncRNAs, m7G-methylated IncRNAs and non-m7G IncRNAs in the HPH and N groups: proportion of each type is shown in the pipe plot. $\mathbf{f}$ and $\mathbf{g}$ Proportion of IncRNAs, m7G-methylated IncRNAs and non-m7G IncRNAs containing different numbers of exons. Up to seven exons are shown. $n=3$ for each group
} 


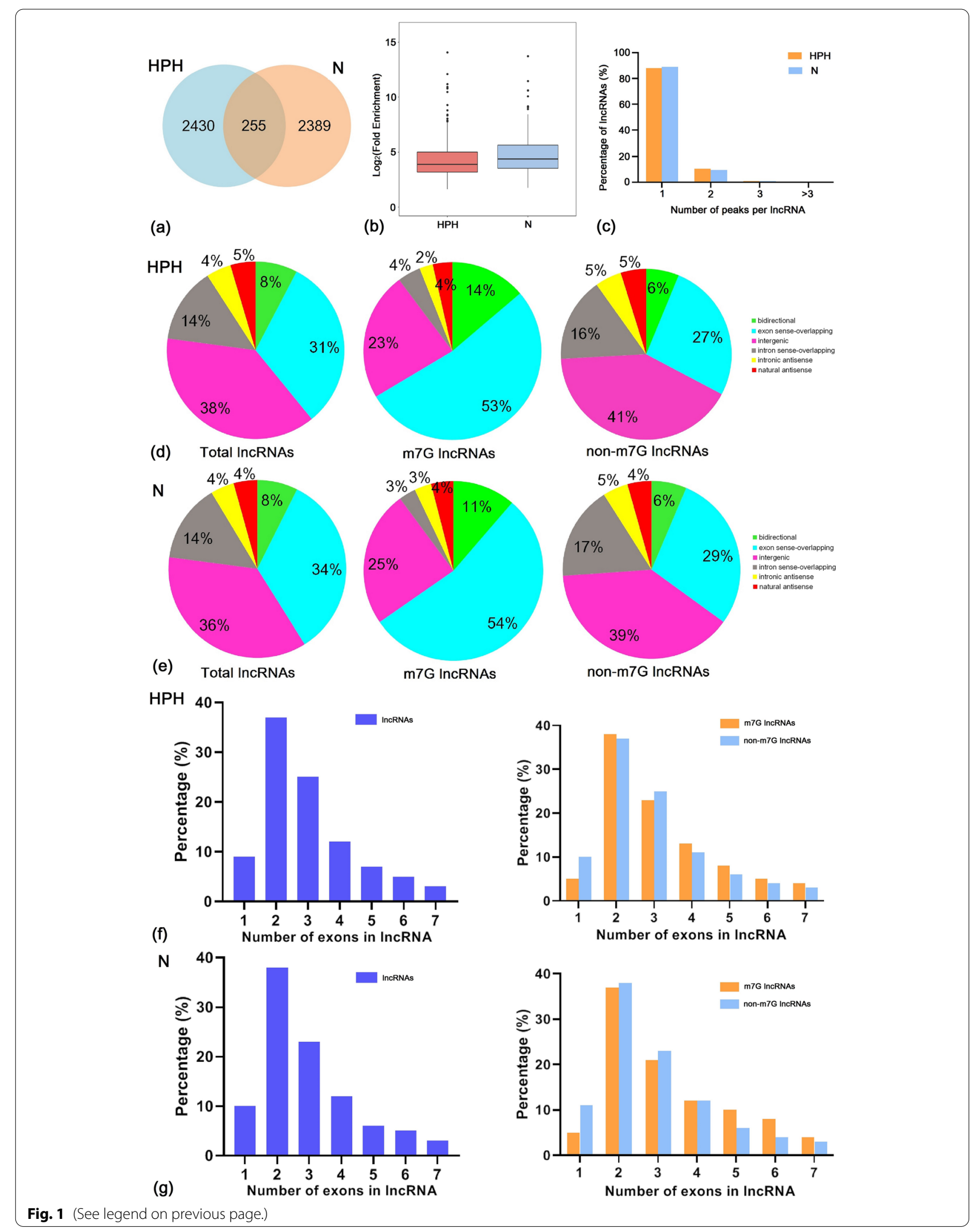




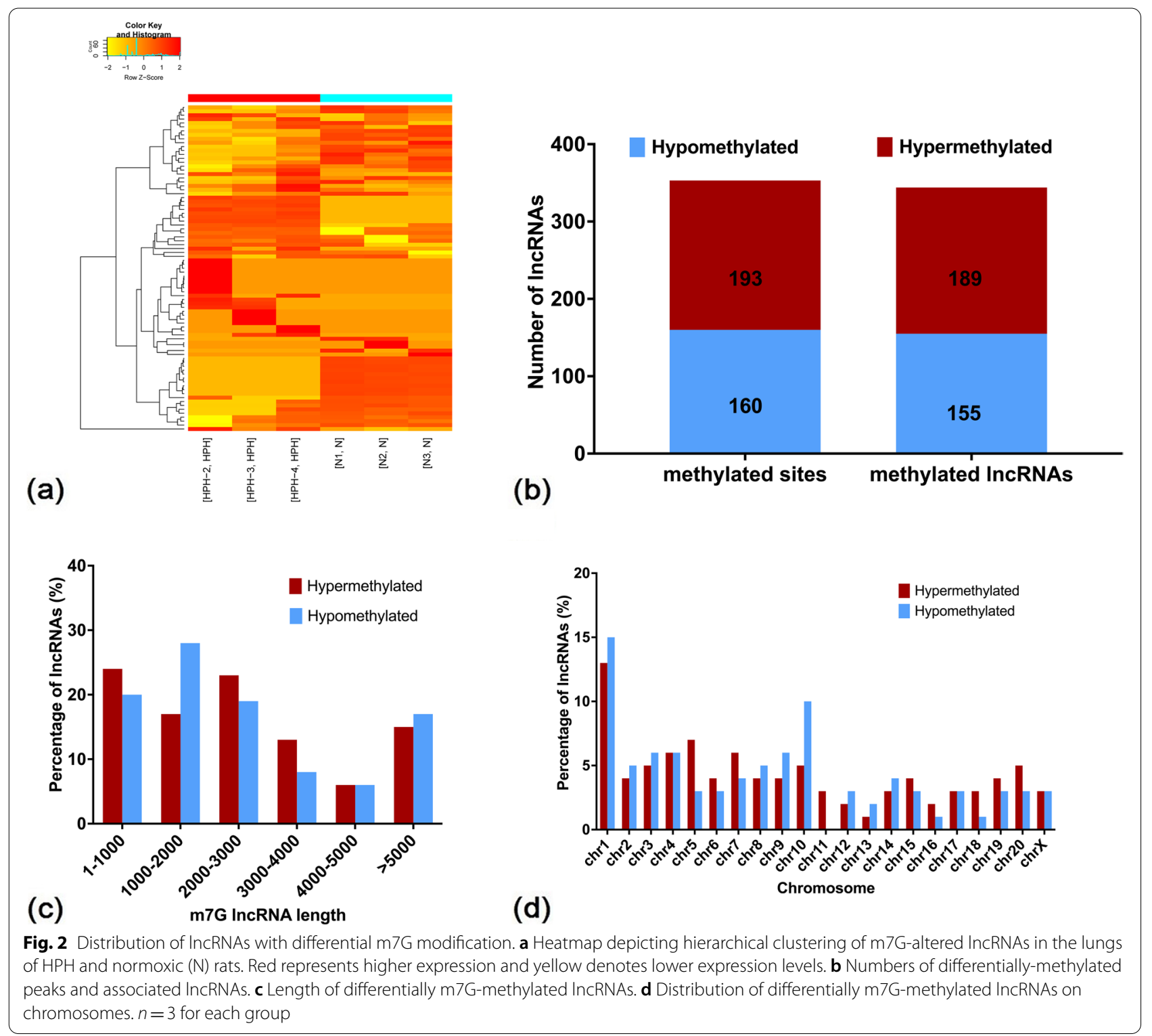

\section{Functional analysis of genes near differentially methylated} IncRNAs

To elucidate the role of differentially methylated lncRNAs in the occurrence and development of HPH, GO and KEGG pathway analyses of genes located near differentially methylated lncRNAs were performed.

In the biological process (BP) category, GO results revealed that genes near hypermethylated $\mathrm{m} 7 \mathrm{G}$ lncRNAs primarily participated in complement activation, negative regulation of smooth muscle contraction and the protein activation cascade. In the cellular component $(\mathrm{CC})$ category, genes were primarily associated with the neuronal cell body, intracellular cellular component and postsynaptic membrane. In terms of molecular function (MF), genes were primarily involved in binding, particularly metal and calcium ion (Fig. 3a). Genes located near hypomethylated lncRNAs were primarily involved in the following BPs: protein O-linked glycosylation, muscle cell differentiation and immunoglobulin secretion. In terms of $\mathrm{CC}$, genes were associated with intracellular organelle, the clathrin vesicle coat and cell cellular component. In terms of MF, genes primarily participated in binding, phosphatidylserine binding and acetylgalactosamine transferase activity (Fig. 3b).

KEGG pathway analysis revealed the four most important signalling pathways associated with genes located near hypermethylated lncRNAs were associated 


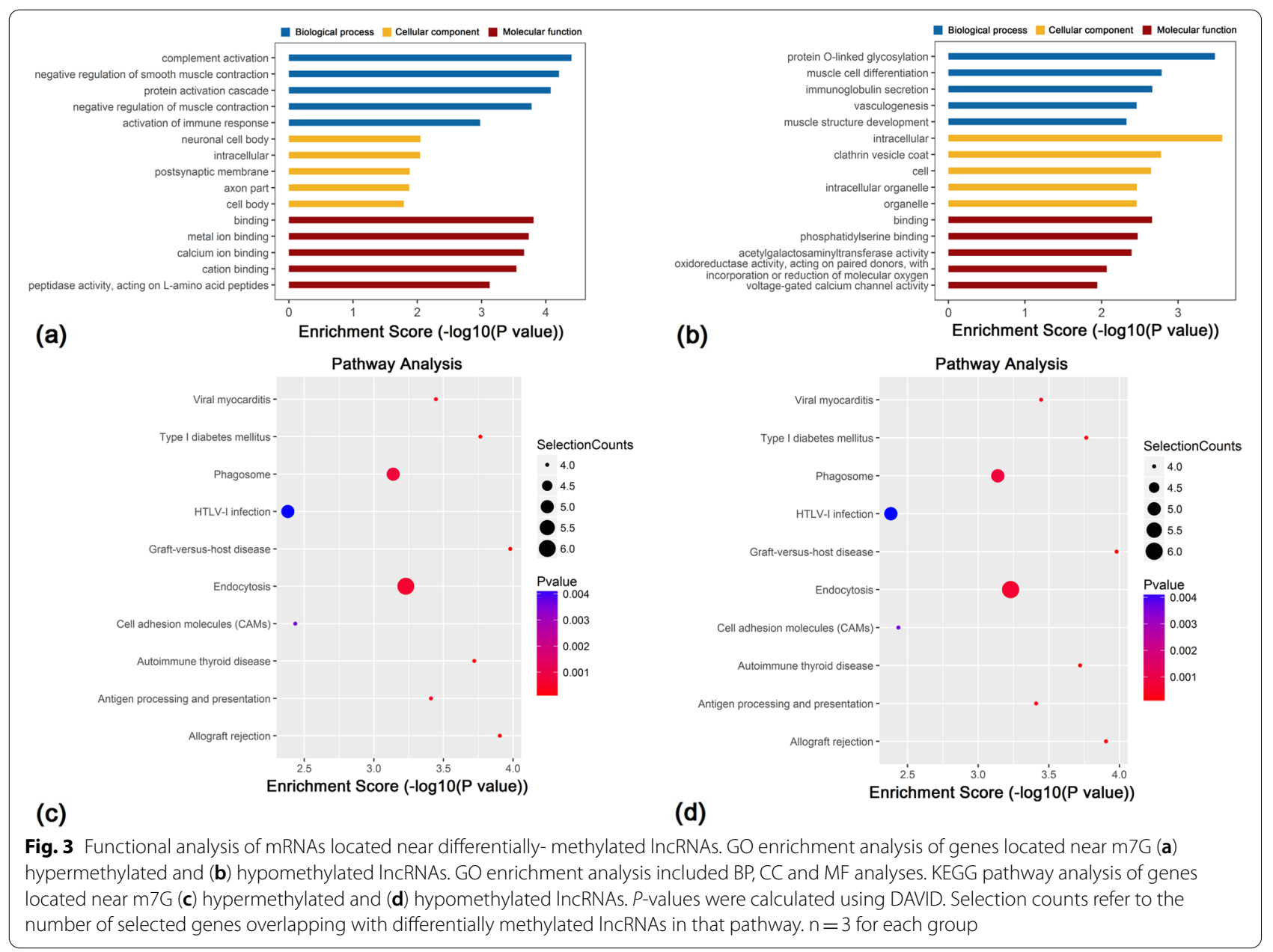

with Staphylococcus aureus infection, the cGMPPKG signalling pathway, complement and coagulation cascades, and Fc gamma R-mediated phagocytosis (Fig. 3c). The four most important signalling pathways for genes located near hypomethylated lncRNAs were associated with graft-versus-host disease, type 1 diabetes mellitus, autoimmune thyroid disease and viral myocarditis (Fig. 3d).

These results suggested that m7G-modified lncRNAs may affect the occurrence and development of $\mathrm{HPH}$ via BPs, cellular composition, MF and signalling pathways.

\section{RNA-seq analysis of differentially expressed IncRNAs in $\mathrm{HPH}$}

Figure 4a shows a heatmap of RNA-seq data in the HPH and control groups, indicating the differential expression of lncRNAs in the two groups. A total of 111 lncRNAs were differentially expressed in $\mathrm{HPH}$, including 90 upregulated and 21 down-regulated types (Fig. 4b).

To further analyse the effects of genes located near differentially expressed lncRNAs, GO enrichment and
KEGG pathways analysis of genes near lncRNAs were performed. GO analysis revealed that genes located near up-regulated lncRNAs were significantly enriched in terms of positive regulation of immune effector processes, catalytic step 2 spliceosome and unfolded protein binding (Fig. 4c). Genes near down-regulated lncRNAs were significantly associated with blastocyst formation, lysozyme activity and intrinsic components of organelle membranes (Fig. 4d).

KEGG pathway analysis revealed that genes near upregulated lncRNAs were primarily involved in antigen processing and presentation, endocytosis, and phagocytosis (Fig. 4e). Genes located near down-regulated lncRNAs were mainly involved in the Wnt signalling pathway. Together, these results indicated that genes near differentially expressed lncRNAs may be related to HPH (Fig. 4f).

\section{Association of $\mathrm{m7G}$ methylation and the expression of IncRNAs}

A total of 972 m7G lncRNAs were identified in the HPH group and $918 \mathrm{~m} 7 \mathrm{G}$ lncRNAs were identified in the 


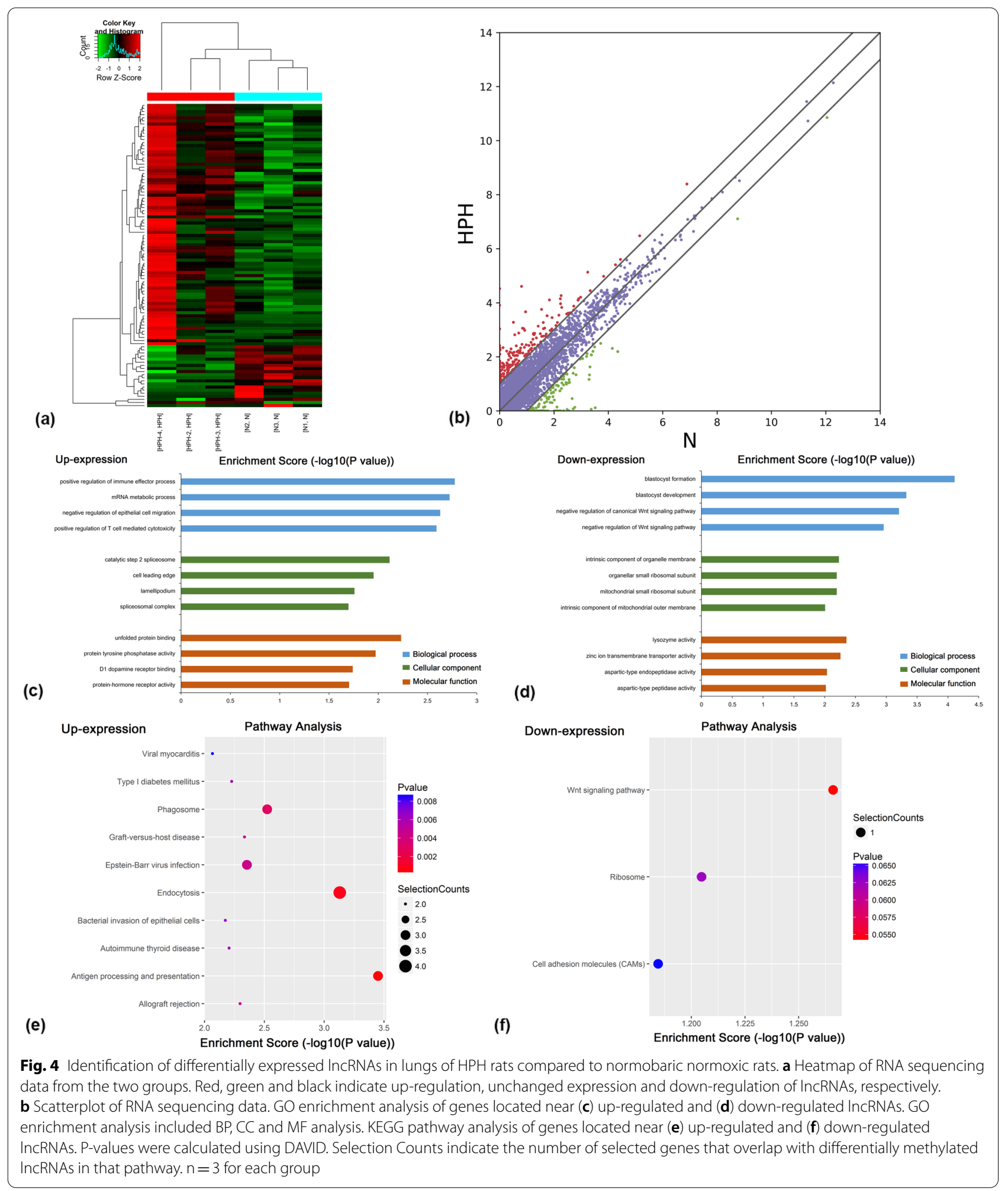

control group. Among these, 767 m7G-modified lncRNAs were detected in both groups (Fig. 5a). The expression of 767 common m7G lncRNAs was investigated to analyse the effect of hypoxia-regulated $\mathrm{m} 7 \mathrm{G}$ methylation. As shown in Fig. 5b, 189 hyper-methylated and 155 hypo-methylated m7G lncRNAs were found in the HPH 


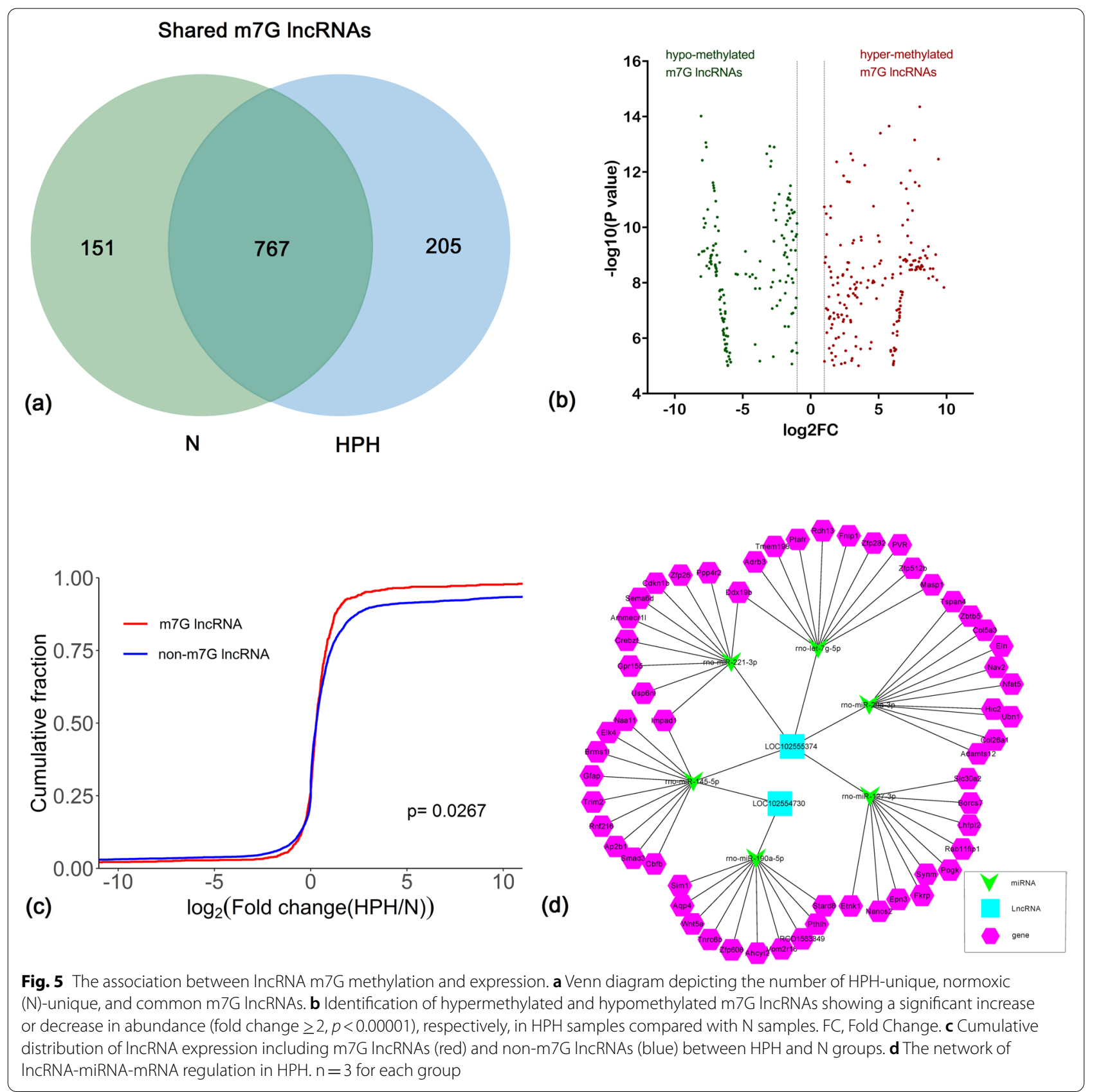

group compared with the control group (fold change $\geq 2$, $P<0.0001)$.

To further determine whether $\mathrm{m} 7 \mathrm{G}$ methylation affected lncRNA expression, all the expressed lncRNAs were divided into $\mathrm{m} 7 \mathrm{G}$ and non-m7G categories. The log two-fold change $(\log 2 \mathrm{FC})$ values of these lncRNAs were determined and a cumulative curve was generated. The proportion of $\mathrm{m} 7 \mathrm{G}$-modified lncRNAs was larger than that of non-m7G lncRNAs. These results indicated that
m7G significantly up-regulated the expression of lncRNAs under hypoxic conditions (Fig. 5c, $P=0.0267$ ).

The combined results of methylation sequencing and RNA sequencing revealed that only two up-regulated lncRNAs were hypermethylated, which originated from chr4: 7450738-7,450,981 (LOC102554730, XR_591973) and chr13: 95420341-95,420,800 (LOC102555374, XR_592398). However, no down-regulated lncRNAs were hypomethylated. 
Table 1 List of downstream target genes of differentially expressed m7G IncRNAs via ceRNA network analysis

\begin{tabular}{lll}
\hline IncRNA & miRNAs & Target genes \\
\hline LOC102555374 & rno-let-7g-5p & Ptafr, Masp1, Tmem198b, Fnip1, Zfp512b, Zfp282, PVR, Ddx19b, Rdh13, Adrb3 \\
LOC102555374 & rno-miR-127-3p & Etnk1, Borcs7, Lhfpl2, Synm, Fkrp, Epn3, Slc30a2, Pogk, Rab1 1fip1, Nanos2 \\
LOC102555374 & rno-miR-221-3p & Ddx19b, Impad1, Crebzf, Gpr155, Zfp26, Usp6n1, Ammecr11, Sema6d, Ppp4r2, Cdkn1b \\
LOC102555374 & rno-miR-29a-3p & Adamts12, Col26a1, Zbtb5, Nfat5, Nav2,Hic2, Col5a3, Eln, Tspan4, Ubn1 \\
LOC102555374 & rno-miR-145-5p & Trim2, Ap2b1, Brms11, Gfap, Cbfb, Rnf216, Impad1, Naa11, Smad3, Elk4 \\
LOC102554730 & rno-miR-190a-5p & Tnrc6b, Vom2r18, RGD1563349, Aqp4, Wnt5a, Ahcyl2, Sim1, Stard8, Pth1h, Zfp608 \\
LOC102554730 &
\end{tabular}

\section{Construction of IncRNA-miRNA-mRNA competing endogenous RNA (ceRNA) network and identification of differentially expressed $\mathrm{m} 7 \mathrm{G}$ IncRNAs}

To explore the potential mRNAs, which were regulated by the lncRNAs, lncXR_592398 and lncXR_592398 were used to build a ceRNA network. Among the predicted miRNAs, miR-190a-5p, miR-145-5p, miR-29a-3p, let-7g-5p, miR-127-3p and miR-221-3p were selected to construct the ceRNA network because they were related to $\mathrm{PH}$ [23]. The top 10 genes for each of these miRNAs were predicted using MirTarget and Miranda programs (Table 1). Thus, the network consisted of PH-associated miRNAs combined with the two differentially expressed m7G lncRNAs and the top 10 mRNAs that bound to the miRNAs, including two lncRNAs, six miRNAs and 58 mRNAs. This ceRNA network clearly suggests that lncRNAs regulated miRNAs and mRNAs. For example, LOC102555374 (XR_592398) bound to rno-let-7g-5p to regulate Tmem198 expression (Fig. 5d).

\section{Up-regulation of m7G XR_591973 and m7G XR_592398 in hypoxic PASMCs}

To further corroborate the results, the expression of the two differentially expressed $\mathrm{m} 7 \mathrm{G}$ lncRNAs in PASMCs treated with hypoxia $\left(1 \% \mathrm{O}_{2}\right)$ and normoxia $\left(21 \% \mathrm{O}_{2}\right)$, respectively, was validated. Using cDNA from the PASMCs as templates, lncXR_591973 and lncXR_592398 were amplified by divergent primers. MeRIP-qRT-PCR was used to determine whether lncXR_591973 and lncXR_592398 were modified by m7G. As shown in Fig. 6, the levels of $\mathrm{m7G}$ lncXR_591973 and m7G lncXR_592398 were significantly increased in hypoxic PASMCs $(P=0.001$ and $P=0.002$, respectively), demonstrating the reliability of the transcriptome-wide m7G-MeRIP-seq analysis.

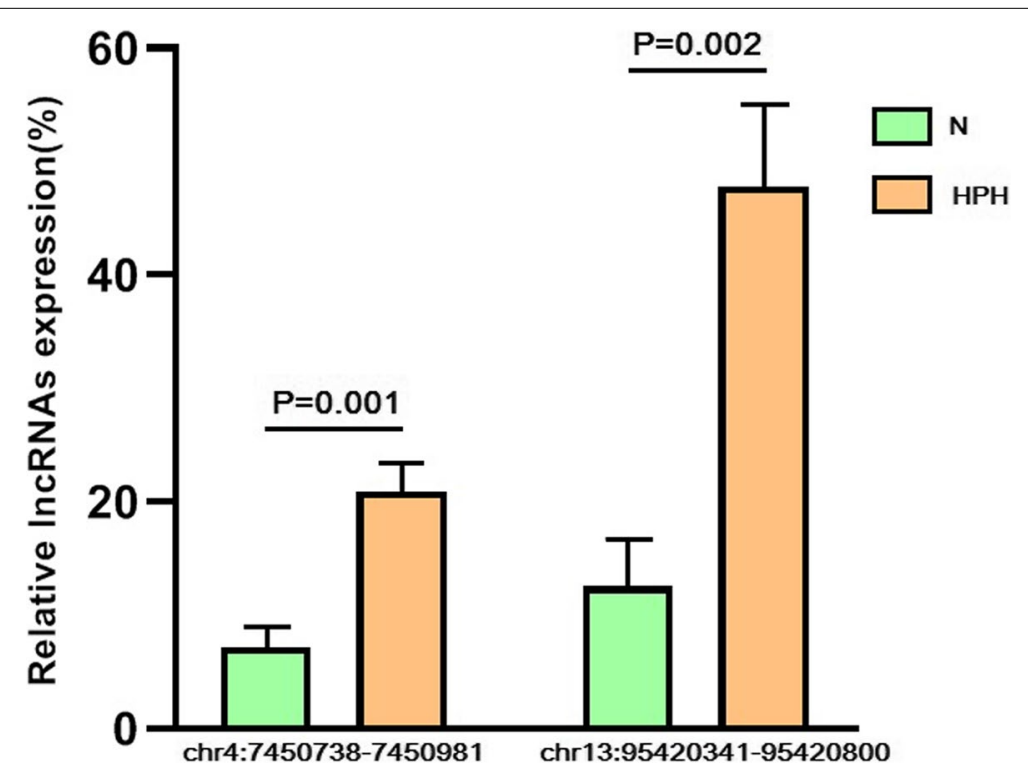

Fig. 6 The expression of m7G IncXR_591973 and m7G IncXR_592398 in PASMCs in hypoxia. MeRIP-qRT-PCR detection of differentially expressed m7G IncRNAs. qRT-PCR was performed after MeRIP in PASMCs exposed to $21 \%(\mathrm{~N})$ or $1 \% \mathrm{O}_{2}(\mathrm{H})$ for $48 \mathrm{~h}$. The expression of each $\mathrm{m7G}$ IncRNA was calculated as the ratio of the anti-m7G level (IP) relative to the control level (input). Data are expressed as the mean $\pm S D$ ( $n=3$ in each group). The P-value was determined using a two-sided paired t-test 


\section{Discussion}

In the present study, transcriptome-wide mapping of m7G lncRNAs in HPH was performed for the first time to elucidate the role of $\mathrm{m} 7 \mathrm{G}$ lncRNAs in the pathogenesis of $\mathrm{PH}$. The m7G modification pattern in HPH samples was distinct from that of normal controls, with a tendency towards lower total m7A abundance in the $\mathrm{HPH}$ group. In addition, the two groups shared only $5 \%$ $\mathrm{m} 7 \mathrm{G}$ peaks. A recent study found that internal m7G/G levels were quite low (range from $\sim 0.02 \%$ to $\sim 0.05 \%$ ) in human and mouse cell lines within mammalian mRNA, which represent roughly $5-10 \%$ of their respective $\mathrm{m} 6 \mathrm{~A} / \mathrm{A}$ ratios. Further, $\mathrm{m} 7 \mathrm{G}$ was detected internally within mammalian mRNA. Although the m7G MeRIPseq method has been widely used, the method still has limitations in detecting m7G internally [24]. These limitations might result in the minor overlap in $\mathrm{m} 7 \mathrm{G}$ peaks found in this study.

The transcriptome-wide analysis revealed that $\mathrm{m} 7 \mathrm{G}$ modifications were associated with a positive correlation with lncRNA expression in HPH samples. This result was further corroborated by $\mathrm{m} 7 \mathrm{G}$ MeRIP-qRT-PCR assays. Similarly, the abundance of m6A modification has been positively associated with lncRNA expression [25]. Kouzarideset al. recently reported that $\mathrm{m} 7 \mathrm{G}$ modifications promoted human miRNA processing by directly affecting the secondary structure of pri-miRNAs, which suppressed cell migration [26]. Additionally, m7G modification was also demonstrated to be essential for efficient pre-mRNA splicing, translation and protein-RNA interactions [27]. Further, accumulating evidence suggests that $\mathrm{m} 7 \mathrm{G}$ modification is significantly related to the development of multiple diseases, including cancers, infections and stem cell-associated diseases [28].

The KEGG analysis in the present study revealed a significant role of up-regulated $\mathrm{m} 7 \mathrm{G}$ lncRNAs in vascular smooth muscle contraction pathway. Pulmonary vasoconstriction is known to play an important role in the pathogenesis of $\mathrm{HPH}$ and represents one of the factors contributing to increased PASMC contraction [4]. Clinical studies have also shown that vasodilating agents ameliorate $\mathrm{PH}$ and cardiac output of patients diagnosed with $\mathrm{HPH}$, which demonstrates the key role of vasoconstriction in HPH pathophysiology [29, 30]. A previous study further reported that inhibiting PASMC contraction in response to hypoxia significantly decreased the RVP of $\mathrm{PH}$ models [31].

lncRNAs act as ceRNAs to indirectly regulate mRNAs via shared microRNAs, which represents a novel layer of RNA crosstalk and plays a critical role in the development of various diseases [32]. m7G plays a critical role in regulating RNA processing, metabolism and function [28], suggesting that
m7G also regulates the lncRNA-miRNA-mRNA co-expression network. Analysis of the differentially expressed $\mathrm{m} 7 \mathrm{G}$ lncRNAs revealed an overlap of 11 pseudogenes between the identified lncRNAs. Among the overlapped pseudogenes, Smad3 is a key downstream element in transforming growth factor (TGF)- $\beta$ signaling pathways [33]. In recent years, the critical role of TGF- $\beta$ signaling pathways in $\mathrm{PH}$ has been better elucidated. Enhanced TGF- $\beta$ signaling has been associated with $\mathrm{PH}$ accompanied by smooth muscle hypertrophy, perivascular fibrosis, and extracellular matrix remodeling [34]. Another overlapped gene, inositol monophosphatase domain containing $1(\operatorname{Impad} 1)$, a novel sulfotransferase that is normally located in golgi bodies, has received increased attention in cancer, as it converts PAP into AMP in human fibroblasts [35]. Increasing evidence indicates that in cancer, Impad1 acted in the ER-Golgi pathway by altering Golgi-mediated secretion of MMPs, resulting in a pro-invasive and metastatic tumour phenotype [36]. Additionally, Impad1 overexpression was shown to inhibit Complex I activity, reducing ROS production in cancer cells, and promoting tumor cell invasion [37]. As a result, the function of Impad1 is diverse and needs further exploration in HPH. Among the non-overlapped pseudogenes, nuclear factor of activated $\mathrm{T}$ cells 5 (Nfat5), the $\mathrm{Ca} 2+/$ calcineurin sensitive transcription factor, was reported to perpetuate the elevation in cytosolic calcium and promote apoptosis resistance of PASMCs. Increased cytosolic calcium contributes to the contractile, hyperproliferative, and antiapoptotic phenotype of PASMCs, which leads to $\mathrm{PH}$ pathogenesis [38]. However, Wnt5a, a key factor of Wnt signaling pathways, plays a key role in the Wnt signaling pathways in $\mathrm{PAH}$ pathobiology. Evidence suggests that alterations in Wnt pathway activation lead to $\mathrm{PH}$ by preventing proper small vessel regeneration and allowing excessive PASMC growth [39]. In general, these downstream targets regulated by lncRNA-miRNA of interest were mostly enriched in $\mathrm{PH}$-associated TGF- $\beta$, Wnt and Nfat signaling pathways. Our study reported for the first time that $\mathrm{m} 7 \mathrm{G}$ influenced lncRNAs, thus regulating the binding of miRNA, resulting in the activation of TGF- $\beta$, Wnt and Nfat signaling pathways.

The main limitations of this study were the lack of clinical samples and the inability to determine the precise mechanism of hypoxia-induced $\mathrm{m} 7 \mathrm{G}$ modification. Thus, studies focusing on the underlying mechanism of m7G modification, as well as clinical transformation, are required to establish the role of $\mathrm{m} 7 \mathrm{G}$ lncRNAs in the pathogenesis of $\mathrm{HPH}$. 


\section{Conclusion}

This study provides the first $\mathrm{m} 7 \mathrm{G}$ transcriptome-wide map of $\mathrm{HPH}$. It demonstrated that the m7G modification pattern in $\mathrm{HPH}$ samples is distinct from that of controls, with a tendency towards lower total m7A abundance in the $\mathrm{HPH}$ group. Further, two significant m7G lncRNAs were found to be associated with $\mathrm{HPH}$ for the first time, and both of them are validated in PASMC cells. The role of $\mathrm{m} 7 \mathrm{G}$ lncRNAs in HPH and its underlying mechanism require further exploration. It is hoped that this will be the start of studies investigating m7G functions and modification in $\mathrm{HPH}$. In the future, the integrated analysis of transcription and translation studies might contribute to the elucidation of the mechanism of $\mathrm{HPH}$. Additional evidence derived from large patient populations with $\mathrm{HPH}$ will improve the value of this study and identify specific $\mathrm{HPH}$-related m7G methylation sites.

\section{Methods}

\section{Establishment of HPH rat model}

4-week male Sprague-Dawley (SD) rats weighing 180 to $200 \mathrm{~g}$ each were used in this study and were supplied by the Animal Experimental Centre, Zhejiang University, China. Rats were randomly allocated to the $\mathrm{HPH}$ and the control group ( $n=6$ in each group). The rat model for this study was established based on our previous study [22], and the same animal group was used in the two studies. The construction and subsequent verification of a hypoxia-induced $\mathrm{PH}$ rat model has been described in our previous study [22]. All rats were sacrificed by exposure to $100 \% \mathrm{CO}_{2}$ in a confined and transparent euthanasia device, and heart and lung tissues were removed for the following experiments. All protocols and procedures were approved by the Ethics Committee of Zhejiang University, China and were conducted in accordance with the guidelines of the National Institutes of Health on the care and use of animals.

\section{Isolation and hypoxia treatment of primary PASMCs}

The isolation of primary PASMCs from rat pulmonary artery was performed as described previously [17]. PASMCs were cultured in Dulbecco's modified Eagle medium supplemented with $10 \%$ foetal bovine serum (FBS). Before experimental use, the cells were incubated under hypoxic $\left(1 \% \mathrm{O}_{2}\right)$ or normoxic $\left(21 \% \mathrm{O}_{2}\right)$ conditions at $37^{\circ} \mathrm{C}$ for $48 \mathrm{~h}$. PASMCs were grown to no more than $90 \%$ confluence and used between passages four and seven.

\section{RNA isolation and RNA-seq analysis}

Total RNA from lungs (1 g) of control and HPH rats was extracted with TRIzol reagent (Invitrogen, CA, USA). The extracted RNA was purified using a NEBNext rRNA
Depletion Kit (New England Biolabs, MA, USA). Next, RNA libraries were prepared using the NEBNext Ultra Directional RNA Library Prep kit (New England Biolabs, MA, USA). Library sequencing was performed on an IlluminaHiseq instrument with $150 \mathrm{bp}$ paired-end reads.

\section{MeRIP and m7G-seq analysis}

The isolated RNA was subjected to immunoprecipitation using the m7G-IPKit (GenSeq, Beijing, China) according to the manufacturer's instructions. RNA was randomly fragmented to about $200 \mathrm{nt}$ using RNA fragmentation reagents (Millipore Sigma, USA). Protein A/G beads (Thermo Fisher Scientific, USA) were coupled to the $\mathrm{m} 7 \mathrm{G}$ antibody via rotation at room temperature for $1 \mathrm{~h}$. The RNA fragments were incubated with the bead-linked antibodies and rotated at $4{ }^{\circ} \mathrm{C}$ for $4 \mathrm{~h}$. Then, the bound RNA was eluted from the beads using Proteinase $\mathrm{K}$ and purified by phenol-chloroform extraction. Purified RNA was used for RNA-seq library generation with a NEBNext ${ }^{\circledR}$ Ultra II Directional RNA Library Prep Kit (New England Biolabs, MA, USA). Paired-end reads were harvested from an IlluminaHiSeq 4000 sequencer.

\section{Construction of a ceRNA network}

The IncRNA-microRNA (miRNA) -mRNA ceRNA network was constructed based on the theory that lncRNA acts as an miRNA sponge to further regulate mRNA. The binding sites of miRNA on differentially expressed $\mathrm{m} 7 \mathrm{G}$ lncRNAs as well as the target mRNA of the miRNA were predicted with Target Scan and miRanda. The miRNAs related to $\mathrm{PH}$ and the top $10 \mathrm{mRNAs}$ binding to each selected miRNA were retained for further network analysis. Pearson correlation analysis was used to assess the ceRNA network construction. Cytoscape v3.6.0 was used to visualise the ceRNA network.

\section{Methylated RNA immunoprecipitation and MeRIP-qPCR} M7G immunoprecipitation (MeRIP) was performed to measure the specific $\mathrm{m} 7 \mathrm{G}$ modification of lncRNA. Total RNA was isolated from hypoxia- and normoxia-treated PASMCs. RNA $(18 \mu \mathrm{g})$ was fragmented to approximately 200 nt-long fragments in an RNA fragmentation buffer. The fragmented RNA was purified using an RNaseMiniElute Kit according to the manufacturer's instructions. Protein A/G beads $(50 \mu \mathrm{L})$ were coupled to $\mathrm{m} 7 \mathrm{G}$ antibody by rotating at room temperature for $30 \mathrm{~min}$. The RNA fragments were then incubated with the bead-linked antibodies and rotated at $4{ }^{\circ} \mathrm{C}$ for $2 \mathrm{~h}$. The immunopurified RNA was purified and first-strand cDNA synthesis was performed by real-time quantitative PCR (QPCR). All the samples were run with the qPCR SYBR Green Master Mix (CloudSeq). The primers used for the detection of $\mathrm{m} 7 \mathrm{G}$ enriched lncRNA are presented in Table 2. 
Table 2 Primer sequences used in the qPCR analysis

\begin{tabular}{ll}
\hline Gene (GenBank) & Primer sequence $\mathbf{( 5}^{\prime} \mathbf{- 3}^{\prime}$ ) \\
\hline chr4:7450738-7,450,981 & Forward GTGATGTGGAAGGGAGCACT \\
& Reverse TGTCTGCCTCTCCGTCTTCT \\
chr13:95420341-95,420,800 & Forward AGGACACCAAGGGAACACTG \\
& Reverse CAGGTACCTCCCAAGCCATA \\
\hline
\end{tabular}

\section{Sequencing data analysis and statistical analysis}

The quality of harvested paired-end reads were controlled by Q30. Cutadapt (v1.9.3) was used for 3' adaptor trimming and the removal of low-quality reads. Clean reads from all libraries were aligned with the reference genome (UCSC RN5) using HISAT2 (v2.0.4). The m7G MeRIPsequencing protocol was performed using methylated sites on RNAs ( $\mathrm{m} 7 \mathrm{G}$ peaks) identified by MACS software. Differentially methylated sites were identified by diffReps and $\mathrm{m} 7 \mathrm{G}$ peaks that overlapped with transcription exons were retained for further study. For lncRNA sequencing, the FPKM (fragments per kilobase of exon per million fragments mapped) value was obtained as the expression profiles of lncRNA using Cuffdiff (v2.2.1). Differentially expressed lncRNAs were identified by the fold change and $p$-value (fold change $\geq 2$ and $P \leq 0.00001$ ). In addition, gene ontology (GO) and kyoto encyclopedia of genes and genomes (KEGG) pathway enrichment analysis of the differentially-expressed RNAs and the differentially-methylated protein-coding genes were performed according to previous reports $[40,41]$. $P$ values are calculated by DAVID tool for GO and KEGG pathway analysis.

Data were presented as the mean \pm standard deviation (SD). Statistical analyses were performed using SPSS 19.0 (Chicago, IL, USA) and GraphPad Prism 5.0 (La Jolla, CA). A two-tailed Student's t-test or two-sided Wilcoxon-Mann-Whiteney test was used to determine the significant differences between the two groups. The number of samples in each group is shown as $n$ in figure legends. Differences of $P<0.05$ were defined as statistically significant. All experiments were independently repeated at least three times.

\footnotetext{
Abbreviations

IncRNAs: Long non-coding RNAs; HPH: Hypoxic pulmonary hypertension; m7G: N7-methylguanosine; PH: Pulmonary hypertension; COPD: Chronic obstructive pulmonary disease; PAECs: Pulmonary artery endothelial cells; PASMCs: pulmonary artery smooth muscle cells; BP: Biological process; CC: Cellular component; MF: Molecular function; SD: Sprague-Dawley; FBS: Foetal bovine serum; miRNA: microRNA; SD: Standard deviation; GO: Gene ontology; KEGG: Kyoto encyclopedia of genes and genomes; Wnt5a: Wnt family member 5a; RGD1563349: Arginine-glycine-aspartic acid1563349; Vom2r18: vomeronasal 2 receptor, 18; Zfp512b, Zfp282,Zfp608, Zfp26: zinc finger protein 512b, 282, 608 and 26; Smad3: SMAD family member 3; Impad1: Inositol monophosphatase domain containing 1; Ap2b1: Adaptor related protein complex 2 subunit beta 1; Cbfb: Core-binding factor subunit beta; Naa11:
}

$\mathrm{N}$-alpha-acetyltransferase 11; Brms11: Breast cancer metastasis-suppressor 1-like; Tspan4: Tetraspanin 4; Usp6nl: USP6 N-terminal like; Pthlh: Parathyroid hormone like hormone; Col5a3: Collagen type $V$ alpha 3 chain; Adrb3: Adrenoceptor beta 3; Nfat5: Nuclear factor of activated T cells 5; Nanos2: Nanos C2HC-type zinc finger 2; Sema6d: Semaphorin 6D; Tnrc6b: Trinucleotide repeat containing adaptor 6B; Slc30a2: Solute carrier family 30 member 2; Masp1: MBL associated serine protease 1; Fkrp: Fukutin related protein; Rnf216: Ring finger protein 216; Epn3: Epsin 3; Stard8: StAR related lipid transfer domain containing 8; Synm: Synemin; Hic2: HIC ZBTB transcriptional repressor 2; Cdkn1b: cyclin dependent kinase inhibitor 1B; Rdh13: Retinol dehydrogenase 13; Ammecr1l: AMME chromosomal region gene 1-like; PVR: Poliovirus receptor; Pogk: Pogo transposable element derived with KRAB domain; Zbtb5: Zinc finger and BTB domain containing 5; Sim1: Single-minded family bHLH transcription factor 1; Gfap: Glial fibrillary acidic protein; Trim2: Tripartite motif containing 2; Borcs7: BLOC-1 related complex subunit 7; Ahcyl2: Adenosylhomocysteinase like 2; Ptafr: Platelet-activating factor receptor; Fnip1: Folliculin interacting protein 1; Gpr155: G protein-coupled receptor 155; Adamts12: A disintegrin-like and metallopeptidase (reprolysin type) with thrombospondin type 1 motif, 12; Ubn1: ubinuclein 1; Rab11fip1: RAB11 family interacting protein 1; Tmem198b: Transmembrane protein 198b; Aqp4: Aquaporin 4; Crebzf: CREB/ATF bZIP transcription factor; Lhfpl2: lipoma HMGIC fusion partner-like 2; Etnk1: Ethanolamine kinase 1; Col26a1: Collagen type XXVI alpha 1 chain; Ppp4r2: Protein phosphatase 4 regulatory subunit 2; Ddx19b: DEAD-box helicase 19B; Eln: Elastin; Nav2: Neuron navigator 2.

\section{Supplementary Information}

The online version contains supplementary material available at https://doi. org/10.1186/s12864-021-08188-8.

Additional file 1: Data S1: m7G methylated sites of IncRNAs in HPH and control samples.

Additional file 2: Data S2: Differentially expressed m7 Gmethylated sites of IncRNAs in the HPH rat model compared to the control rat model.

\section{Acknowledgements}

The authors gratefully appreciate Cloud-Seq Biotech Ltd. Co. (Shanghai, China) for the MeRIP-Seq service and the subsequent bioinformatics analysis.

\section{Authors' contributions}

H.W. and RB.C. performed the experiments, analysed the data, drafted the manuscript, and created the figures; SN.Z. analysed the results and generated the figures; RF.Z. designed the study, analysed the data, explained the findings and wrote the paper. All authors read and approved the final manuscript.

\section{Funding}

This study was supported by the National Natural Science Foundation of China (Grant Numbers 81570043 and 82170063), the Science Foundation of Ministry of Health of Jiangsu Province in China (Grant Number ZDA2020016) and the 2021 Jiangsu Shuangchuang (Mass Innovation and Entrepreneurship) Talent Program (Grant Number JSSCBS20210147). The funders had no role in study design, data collection and analysis, decision to publish, or preparation of the manuscript.

\section{Availability of data and materials}

The raw high-throughput $\mathrm{m} 7 \mathrm{G}$ and IncRNAs sequencing generated during the current study can be obtained from the NCBI Gene Expression Omnibus (GEO): accession number GSE182042 and GSE182054, [https://www.ncbi.nlm. nih.gov/geo/query/acc.cgi?acc=GSE182042], token:[klsluyqezjkfdir]. [https://www.ncbi.nlm.nih.gov/geo/query/acc.cgi?acc=GSE182054], token:[kvmxqquixvszrmj].

\section{Declarations}

Ethics approval and consent to participate

The study protocol has been submitted to and approved by the Institutional Animal Care and Use Committee of Zhejiang University, China (permit number: SYXK 2017-0006). In addition, the study is reported in accordance with ARRIVE guidelines (https://arriveguidelines.org). 


\section{Consent for publication \\ Not applicable.}

\section{Competing interests}

The authors declare that they have no competing interests.

\section{Author details}

'Department of Respiratory Medicine, Zhongda Hospital of Southeast University, 87 Dingjiaqiao Road, Nanjing 210009, People's Republic of China. ${ }^{2}$ Department of Radiology, Sir Run Run Shaw Hospital, Zhejiang University School of Medicine, Hangzhou 310000, People's Republic of China.

Received: 19 July 2021 Accepted: 17 November 2021

Published online: 07 January 2022

\section{References}

1. Frost A, Badesch D, Gibbs J, et al. Diagnosis of pulmonary hypertension. Eur Respir J. 2019;53(1):1801904.

2. Simonneau G, Montani D, Celermajer DS, et al. Haemodynamic definitions and updated clinical classification of pulmonary hypertension. Eur Respir J. 2019;53(1):1801913.

3. Rowan SC, Keane MP, Gaine S, et al. Hypoxic pulmonary hypertension in chronic lung diseases: novel vasoconstrictor pathways. Lancet Respir Med. 2016:4:225-36.

4. Kylhammar D, Rådegran G. The principal pathways involved in the in vivo modulation of hypoxic pulmonary vasoconstriction, pulmonary arterial remodelling and pulmonary hypertension. Acta Physiol (Oxf). 2017;219:728-56.

5. Ransohoff JD, Wei Y, Khavari PA. The functions and unique features of long intergenic non-coding RNA. Nat Rev Mol Cell Biol. 2018;19:143-57.

6. Simion V, Haemmig S, Feinberg MW. LncRNAs in vascular biology and disease. Vasc Pharmacol. 2019;114:145-56.

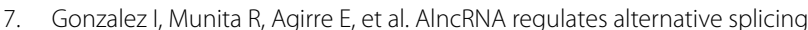
via establishment of a splicing-specific chromatin signature. Nat StructMol Biol. 2015:22:370-6.

8. Liu Y, Sun Z, Zhu J, et al. LncRNA-TCONS_00034812 in cell proliferation and apoptosis of pulmonary artery smooth muscle cells and its mechanism. J Cell Physiol. 2018;233:4801-14.

9. Derrien $T$, Johnson R, Bussotti G, et al. The GENCODE v7 catalog of human long noncoding RNAs: analysis of their gene structure, evolution, and expression. Genome Res. 2012;22:1775-89.

10. Fu XD. Non-coding RNA: a new frontier in regulatory biology. Natl Sci Rev. 2014;1:190-204.

11. Huang Y. The novel regulatory role of IncRNA-miRNA-mRNA axis in cardiovascular diseases. J Cell Mol Med. 2018;22:5768-75.

12. Freedman JE, Miano JM. Challenges and opportunities in linking long noncoding RNAs to cardiovascular, lung, and blood diseases. Arterioscler Thromb Vasc Biol. 2017;37:21-5.

13. Kopp F, Mendell JT. Functional classification and experimental dissection of long noncoding RNAs. Cell. 2018;172:393-407.

14. Han B, Bu P, Meng X, et al. Microarray profiling of long noncoding RNAs associated with idiopathic pulmonary arterial hypertension. Exp Ther Med. 2019:18:2353.

15. Zhang CY, Liu M, Wan JM, et al. Role of noncoding RNA in pulmonary arterial hypertension and potential drug therapeutic target. Curr Top Med Chem. 2018;18:975-86.

16. Wang S, Cao W, Gao S, et al. TUG1 regulates pulmonary arterial smooth muscle cell proliferation in pulmonary arterial hypertension. Can J Cardiol. 2019;35:1534-45

17. Su H, Xu X, Yan C, et al. LncRNA H19 promotes the proliferation of pulmonary artery smooth muscle cells through AT(1)R via sponging let-7b in monocrotaline-induced pulmonary arterial hypertension. Respir Res. 2018;19:254.

18. He C. Grand challenge commentary: RNA epigenetics. Nat Chem Biol. 2010;6:863-5

19. Jühling F, Mörl M, Hartmann RK, et al. tRNAdb 2009: compilation of tRNA sequences and tRNA genes. Nucleic Acids Res. 2009;37:D159-62.

20. Tomikawa C. 7-Methylguanosine modifications in transfer RNA (tRNA). Int J Mol Sci. 2018;19(12):4080.
21. Furuichi Y. Discovery of $m(7) G$-cap in eukaryotic mRNAs. Proc Jpn Acad Ser B Phys Biol Sci. 2015;91:394-409.

22. Su H, Wang G, Wu L, et al. Transcriptome-wide map of m(6)a circRNAs identified in a rat model of hypoxia mediated pulmonary hypertension. BMC Genomics. 2020;21:39.

23. Caruso $P$, MacLean MR, Khanin R, et al. Dynamic changes in lung microRNA profiles during the development of pulmonary hypertension due to chronic hypoxia and monocrotaline. Arterioscler Thromb Vasc Biol. 2010;30:716-23.

24. Zhang LS, Liu C, Ma H, et al. Transcriptome-wide mapping of internal N(7)-MethylguanosineMethylome in mammalian mRNA. Mol Cell. 2019;74:1304-16.e8.

25. Wu Y, Yang $X$, Chen Z, et al. m6A-induced IncRNA RP11 triggers the dissemination of colorectal cancer cells via upregulation of Zeb1. Mol Cancer. 2019;18:87.

26. Pandolfini L, Barbieri I, Bannister AJ, et al. METTL1 promotes let-7 MicroRNA processing via m7G methylation. Mol Cell. 2019;74:1278-90.e9.

27. Chu JM, Ye TT, Ma CJ, et al. Existence of internal N7-Methylguanosine modification in mRNA determined by differential enzyme treatment coupled with mass spectrometry analysis. ACS Chem Biol. 2018;13:3243-50.

28. Malbec L, Zhang T, Chen YS, et al. Dynamic methylome of internal mRNA N7-methylguanosine and its regulatory role in translation. Cell Res. 2019;29:927-41.

29. Blanco I, Santos S, Gea J, et al. Sildenafil to improve respiratory rehabilitation outcomes in COPD: a controlled trial. Eur Respir J. 2013;42:982-92.

30. Hoeper MM, Halank M, Wilkens $H$, et al. Riociguat for interstitial lung disease and pulmonary hypertension: a pilot trial. Eur Respir J. 2013;41:853-60.

31. Lee SJ, Zhang M, Hu K, et al. CCN1 suppresses pulmonary vascular smooth muscle contraction in response to hypoxia. Pulm Circ. 2015:5:716-22.

32. Braga EA, Fridman MV, Moscovtsev AA, et al. LncRNAs in ovarian Cancer progression, metastasis, and Main pathways: ceRNA and alternative mechanisms. Int J Mol Sci. 2020;21(22):8855.

33. Sanada TJ, Sun $X Q$, Happé $C$, et al. Altered TGFB/SMAD signaling in human and rat models of pulmonary hypertension: an old target needs attention. Cells. 2021;10(1):84.

34. Sharmin N, Nganwuchu CC, Nasim MT. Targeting the TGF- $\beta$ signaling pathway for resolution of pulmonary arterial hypertension. Trends Pharmacol Sci. 2021:42:510-3.

35. Frederick JP, Tafari AT, Wu SM, et al. A role for a lithium-inhibited Golgi nucleotidase in skeletal development and sulfation. Proc Natl Acad Sci U S A. 2008;105:11605-12.

36. Bajaj R, Kundu ST, Grzeskowiak CL, et al. IMPAD1 and KDELR2 drive invasion and metastasis by enhancing Golgi-mediated secretion. Oncogene. 2020:39:5979-94.

37. Yang YF, Wang YY, Hsiao M, et al. IMPAD1 functions as mitochondrial electron transport inhibitor that prevents ROS production and promotes lung cancer metastasis through the AMPK-Notch1-HEY1 pathway. Cancer Lett. 2020;485:27-37.

38. Kang $K$, Peng $X$, Zhang $X$, et al. MicroRNA-124 suppresses the transactivation of nuclear factor of activated $T$ cells by targeting multiple genes and inhibits the proliferation of pulmonary artery smooth muscle cells. J Biol Chem. 2013;288:25414-27.

39. de Jesus PV, Yuan K, Alastalo TP, et al. Targeting the Wnt signaling pathways in pulmonary arterial hypertension. Drug Discov Today. 2014;19:1270-6.

40. Harris MA, Clark J, Ireland A, et al. The gene ontology (GO) database and informatics resource. Nucleic Acids Res. 2004;32(Database issue):D258-61.

41. Kanehisa M, Furumichi M, Sato Y, Ishiguro-Watanabe M, Tanabe M. KEGG: integrating viruses and cellular organisms. Nucleic Acids Res. 2021;49(D1):D545-51.

\section{Publisher's Note}

Springer Nature remains neutral with regard to jurisdictional claims in published maps and institutional affiliations. 